\title{
Mice shed light on OCD
}

An international group of scientists is delving into the genetic basis of obsessive-compulsive disorder (OCD) using mice that are genetically engineered to lack the gene Sapap3, which is involved in neurotransmitter signaling. Deleting Sapap3 resulted in greater anxiety and compulsive grooming to the point of selfinjury in these mice. Both the anxiety and excessive grooming were diminished when the mice were treated with a selective serotonin reuptake inhibitor (SSRI). SSRIs are commonly prescribed as treatment for OCD in humans but are effective only about half the time. The mice's behavior and response to SSRIs may be analogous to symptoms of human OCD.

Guoping Feng of Duke University Medical Center (Durham, NC) led the study, which included collaborators from China and Portugal and was published in Nature (doi:10.1038/nature06105, published online 23 August 2007).

Feng and his colleagues originally deleted the gene in mice as part of an investigation of neuronal communication in the

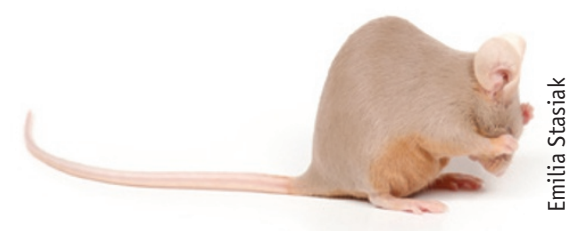

striatum, an area of the brain that controls movement, decision-making and information processing. They noticed that all the Sapap3 mutant mice developed lesions on the head, neck and snout regions. The lesions began as hairless patches below the eyes or swelling of the snout and grew into wounds on both sides of the face covering large areas of the head and neck. The lesions were not caused by other mice or by pre-existing inflammation or skin defects. The mutant mice spent much more time grooming themselves than did wildtype mice without the gene deletion, even grooming themselves during the time when mice normally sleep. The researchers concluded that the mice were grooming themselves so excessively as to cause the lesions. Upon observing this compulsive-like behavior, they looked for and found other OCD-like symptoms in the mice, such as greater anxiety and response to SSRIs.

OCD is a relatively common psychiatric disorder in humans, present in roughly $2 \%$ of us. Its neurological basis is not well understood, but the circuitry in the corticostriato-thalamo-cortical area of the brain is thought to be involved, in addition to genetic factors. OCD is thought to have multiple mechanisms of development. Feng tells $L a b$ Animal, "Previous human studies of OCD have been mostly focused on serotonin and dopamine; our study indicates that defects in neurotransmission by glutamate play an important role in OCD-like behavior. This opens up a new area for research in identifying genes involved in OCD." Feng also noted that this discovery could lead to new treatment strategies targeting glutamate-mediated neurotransmission. Monica Harrington

\section{MEGA MICE}

New genetic research may make cats think twice before they pounce: Se-Jin Lee of Johns Hopkins University School of Medicine (Baltimore, MD) has managed to quadruple mice's muscle mass by turning off one gene and overexpressing another.

Previous research has shown that mice engineered to lack the gene for production of myostatin, a protein that has a critical role in regulating skeletal muscle mass, can grow to double the size of wildtype mice. Deactivation of the myostatin gene is also known to increase muscle mass in cattle, sheep, dogs and humans.

In his present study, Lee added a ligand inhibitor called follistatin to the equation (PLOS ONE 2, e789; 2007). Follistatin is known to block myostatin activity, so it is perhaps not surprising that when Lee engineered myostatin-producing mice to overexpress follistatin, they grew to double the size of wildtype mice. But when mice that lacked the myostatin gene were modified to produce more follistatin, they grew to as large as four times normal size. This suggests that in addition to myostatin, follistatin regulates other proteins that participate in mouse muscle growth. These proteins seem to function differently from myostatin, primarily affecting the size of muscle fibers, whereas myostatin regulates both the size and the number of fibers.
This study may shed light on human muscle growth. In humans, the circulating levels of myostatin in the blood are far lower than those in mice. The role of follistatin in human muscle growth is not known, Lee says, but the importance of this study lies in the discovery that additional ligands may be at work. "It is possible that the other proteins that cooperate with myostatin may play a relatively more important role [than myostatin] in humans," Lee tells Lab Animal. "One of the highest priorities for us is to identify the other proteins." Determining which ligands affect muscle mass will allow researchers to develop strategies for treating human diseases in which muscle growth can be beneficial. These can include muscle degenerative diseases such as muscular dystrophy, or diseases like AIDS and cancer in which patients suffer from muscle loss. Understanding the mechanisms of muscle growth may also help treatment of metabolic diseases such as obesity and type II diabetes: Lee's group previously found that mice lacking myostatin may be protected against fat accumulation and insulin resistance, possibly as a result of their additional skeletal muscle.

Karen Marron 